\title{
QUEEN'S
UNIVERSITY
BELFAST
}

\section{Measurement of electromagnetic pulses generated during interactions of high power lasers with solid targets}

Marco, M. D., Krása, J., Cikhardt, J., Pfeifer, M., Krouský, E., Margarone, D., Ahmed, H., Borghesi, M., Kar, S., Giuffrida, L., Vrana, R., Velyhan, A., Limpouch, J., Korn, G., Weber, S., Velardi, L., Side, D. D., Nassisi, V., \& Ullschmied, J. (2016). Measurement of electromagnetic pulses generated during interactions of high power lasers with solid targets. Journal of Instrumentation, 11, [C06004]. https://doi.org/10.1088/17480221/11/06/C06004

Published in:

Journal of Instrumentation

Document Version:

Peer reviewed version

Queen's University Belfast - Research Portal:

Link to publication record in Queen's University Belfast Research Portal

Publisher rights

(C) 2016 IOP Publishing Ltd and Sissa Medialab srl

This is an author-created, un-copyedited version of an article

accepted for publication in Journal of Instrumentation. The publisher is

not responsible for any errors or omissions in this version of the

manuscript or any version derived from it. The Version of Record is

available online at http://iopscience.iop.org/article/10.1088/1748-0221/11/06/C06004/meta

\section{General rights}

Copyright for the publications made accessible via the Queen's University Belfast Research Portal is retained by the author(s) and / or other copyright owners and it is a condition of accessing these publications that users recognise and abide by the legal requirements associated with these rights.

Take down policy

The Research Portal is Queen's institutional repository that provides access to Queen's research output. Every effort has been made to ensure that content in the Research Portal does not infringe any person's rights, or applicable UK laws. If you discover content in the Research Portal that you believe breaches copyright or violates any law, please contact openaccess@qub.ac.uk. 


\title{
Measuring of electromagnetic pulses generated during interactions of high power lasers with solid targets
}

\author{
M. De Marco ${ }^{a, b, 1}$, J. Krása ${ }^{a}$, J. Cikhardt ${ }^{c, g}$, M. Pfeifer $^{c}$, E. Krouskýc, D. Margarone ${ }^{a}$, \\ H. Ahmed ${ }^{d}$, M. Borghesi ${ }^{d}$, S. Kar ${ }^{d}$, L. Giuffrida ${ }^{a}$, R. Vrana ${ }^{a}$, A. Velyhan ${ }^{a}$, \\ J. Limpouch ${ }^{b}$, G. Korn ${ }^{a}$, S. Weber ${ }^{a}$, L. Velardi ${ }^{e}$, D. Delle Side ${ }^{f}$, V. Nassisi ${ }^{f}$ and J. \\ Ullschmied $^{\mathrm{c}}$ \\ a. Institute of Physics ASCR, v.v.i (FZU), ELI-Beamlines project, \\ Prague, Czech Republic \\ b. Czech Technical University in Prague, FNSPE, \\ Brehavá 7, 11519 Prague 1, Czech Republic \\ c. Institute of Plasma Physics CAS, \\ Za Slovankou 3, 18200 Prague 8, Czech Republic \\ d. School of Mathematics and Physics, Queen's University of Belfast, \\ Belfast BT7 1NN, United Kingdom \\ e. CNR NANOTEC, \\ Via Amendola 122/D, 70126 Bari, Italy \\ f. LEAS, Dipartimento di Matematica e Fisica, Universitá del Salento, \\ Via Arnesano, sn-73100 Lecce, Italy \\ g. Czech Technical University in Prague, FEE, \\ Technická 2, 166 27, Prague 6
}

E-mail: Massimo.DeMarco@eli-beams.eu

ABSTRACT: A target irradiated with a high power laser pulse, blows off a large amount of charge and as a consequence the target itself becomes a power generator of electromagnetic pulses (EMP) owing to high return current flowing to the ground through the target holder. The first measurement of the magnetic field induced by the neutralizing current reaching a value of a few kA was performed with the use of an inductive target probe at the PALS Laser Facility (Cikhardt et al. Rev. Sci. Instrum. 85 (2014) 103507). A full description of EMP generation should contain information on the spatial distribution and temporal variation of the electromagnetic field inside and outside of the interaction chamber. For this reason, we consider the interaction chamber as a resonant cavity in which different modes of EMP oscillate for hundreds of nanoseconds, until the EMP is transmitted outside through the glass windows and EM waves are attenuated. Since the experimental determination of the electromagnetic field distribution is limited by the number of employed antennas, a mapping of the electromagnetic field has to be integrated with numerical simulations. Thus, this work reports on a detailed numerical mapping of the electromagnetic field inside the interaction chamber at the PALS Laser Facility (covering a frequency spectrum from $100 \mathrm{MHz}$ to $3 \mathrm{GHz}$ ) using the commercial code COMSOL Multiphysics 5.2. Moreover we carried out a comparison of the EMP generated in the parallelepiped-like interaction chamber used in the Vulcan Petawatt Laser Facility at the Rutherford Appleton Laboratory, against that produced in the spherical interaction chamber of PALS.

KEYWORDS: High power electromagnetic pulses; Target current; Laser-produced plasmas. 


\section{Contents}

\section{Introduction}

2. Theory, simulations and measurements at PALS

3. EMP comparison between RAL and PALS experiments

\section{Conclusions}

\section{Introduction}

When high power laser pulses interact with solid targets, electromagnetic pulses are produced. This phenomenon is strongly connected with the emission of a large amount of hot electrons from the target, leaving it positively charged [1] which results in the flow of pulsed return current through the stalk holding the target [2]. The target's voltage appears as transient for a time of a microsecond with maximum amplitude in the order of hundreds of $\mathrm{kV}$ and with a broad frequency range from megahertz to hundreds of gigahertz [3]. The generated EMP can couple with laboratory equipment, damaging electronic devices and interfering with their signals. Many efforts were carried out and many ideas were proposed to mitigate the production of EMP in high power laser facilities and to reduce its effects on electronic devices $[4,5]$, as well as to reduce the coupling of EMP with the detected signals such as for the ion collector (IC) ones [6]. To be able to prevent the problem on the electronic, as well as to increase our knowledge on the generation and features of EMP, it is important to map the fields generated around the target. Since the experimental determination of the electromagnetic field distribution is limited by the number of employed antennas, a mapping of such electromagnetic fields should be completed with numerical simulations. In this contribution we focus our attention on the resonant features of the EMP and we report on a first attempt of target chamber mapping by measurements of magnetic fields by two B-probes and simulations using COMSOL Multiphysics 5.2. The EMP detected experimentally at the Rutherford Appleton Laboratory employing Vulcan petawatt laser, and at PALS Laboratory using PALS ns-class and TiSa fsclass lasers, are presented and supported by simulations.

\section{Theory, simulations and measurements at PALS}

The 3-TW iodine laser at PALS Research Centre in Prague was focused with a focal spot diameter of $\sim 80 \mu \mathrm{m}$ for plasma generation at the front side of a thick $\mathrm{Cu}$ target (T). The target chamber was a steel spherical chamber with a radius of $500 \mathrm{~mm}$ (Fig. 1 (a), 1 (b)) with a glass window allowing the laser beam with a diameter of $29 \mathrm{~cm}$ to enter in. An inductive target probe (T-probe) was mounted on the target-target holder system for detection of its current flowing to the ground [2] as well as B-probes were placed in the target chamber in different positions. The B-probes and T-probe were connected with a Tektronix TDS 694C- $3 \mathrm{GHz}, 10 \mathrm{GS} / \mathrm{s}$ oscilloscope by flexible double shielded cables to decrease the level of environmental noise affecting it.

The signals of B-probes last a few hundreds of nanoseconds and at the beginning they show a large positive or negative peak depending on the physical orientation of the probe with respect to the target-target holder system. 
a)

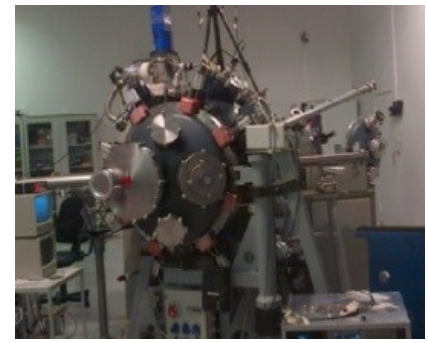

b)

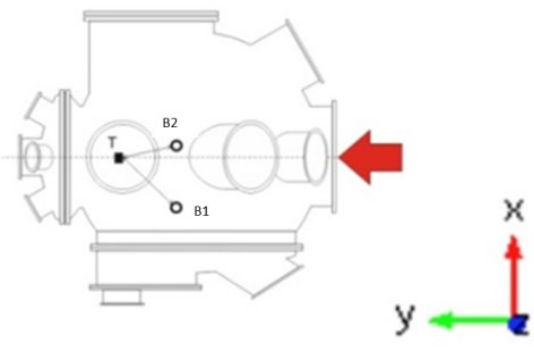

Fig. 1. Picture of target chamber at PALS facility (a), and schematic top view (not in scale) of the target chamber with the applied positioning of B-probes B1 and B2 (b), T denotes target and red arrow marks the incident laser beam.

Detected signals indicate that the temporal profile of the EMP is composed of a fast transient period followed by a longer quasi-stationary one due to the resonant behaviour of EMP inside the target chamber $[6,7,8]$. Since we can assume that the target chamber works as a resonant cavity, it is possible to predict theoretically this behaviour considering it in a first approximation as an ideal resonant cavity. The resonant frequency of a particular mode comes from a proper Maxwell's equation solution satisfying the boundary conditions imposed by the cavity. When the walls of the cavity conduct perfectly, the electric and the magnetic fields must be perpendicular and parallel to the walls, respectively, over the entire surface where these fields are not zero. Using the lumped circuit approximation and the boundary conditions as reported in $[9,10]$, we estimate for the target chamber at PALS facility, the fundamental resonant frequency is $262 \mathrm{MHz}$, as confirmed by simulations.

The COMSOL Multiphysic 5.2 simulation [11] allows us to approximate very well the spatial distribution of the EM field resonating inside the PALS interaction chamber, however it is necessary to build a faithful geometry, while adding at least the main components such as the focussing lens, its metallic holder, metallic plate and the glass window, as well as all the boundary conditions must be fixed by the physical parameters in order to get a model as closer to the reality as possible.

The simulation was based on the following equations of the oscillating wave inside the cavity (1) and of the impedance boundary conditions (2):

$$
\nabla X \mu_{r}^{-1}(\nabla X \boldsymbol{E})-k_{0}^{2}\left(\varepsilon_{r}-\frac{j \sigma}{\delta \varepsilon_{0}}\right) \boldsymbol{E}=0
$$

$$
\sqrt{\frac{\mu_{0} \mu_{r}}{\varepsilon_{0} \varepsilon_{r}-j \sigma / \delta}} \boldsymbol{n} X \boldsymbol{H}+\boldsymbol{E}-(\boldsymbol{n} \cdot \boldsymbol{E}) \boldsymbol{n}=0,
$$

where $\mu_{r}$ is the relative magnetic permeability, $\mu_{0}$ the magnetic permeability in vacuum, $\boldsymbol{E}$ the electric field vector, $k_{0}$ the wave number, $j$ the imaginary unit, $\sigma$ the electrical conductivity, $\varepsilon_{r}$ relative permittivity, $\varepsilon_{0}$ permittivity in vacuum, $\boldsymbol{n}$ the unit vector perpendicular to the inside surface of the cavity (in this case the target chamber) and $\delta$ is the imaginary part of the complex angular frequency $s=\omega-j \delta$.

The spatial distribution of the magnetic field inside a spherical cavity free of metal and glass objects is shown in Fig. $2 \mathrm{~b}$ and the perturbed case by inserting the main items in, is shown in Fig. 2c. We want to stress that in this kind of simulation we get meaningful results only about 
the spatial distribution and not about the strength of the fields at different frequencies. The more realistic chamber structure causes a change in the magnetic field pattern compared to the ideal one shown in Fig. 2a and resonates at shifted frequencies as for example $287 \mathrm{MHz}$. Fig. 3 shows in more detail the distribution of the magnetic field flux density at this frequency. The simulation of the spatial distribution of the magnetic flux density in the target chamber demonstrates that the detected EMP strongly depends on the position and orientation of the Bprobe inside the interaction chamber.

a)

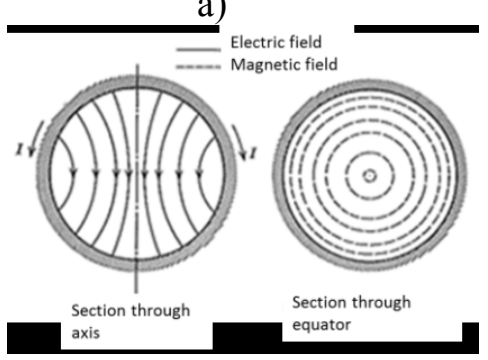

b)

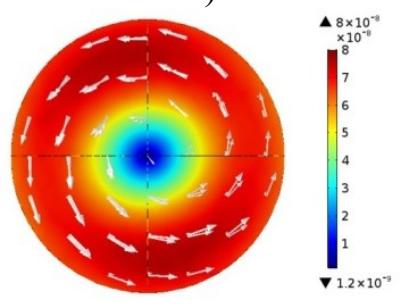

c)

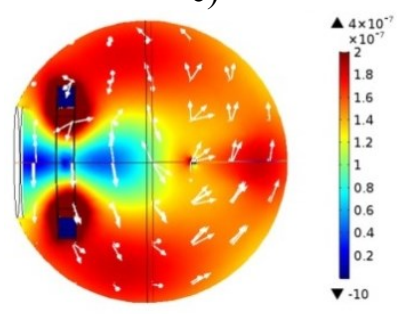

Fig. 2. (a) The fundamental TM mode in an ideal spherical resonant cavity [7]; (b) The magnetic field of the simulated fundamental TM mode; the arrows indicate the magnetic field flux. (c) A distortion of the magnetic field in the target chamber filled by the metal plate, lens, metallic lens holder and window.

a)

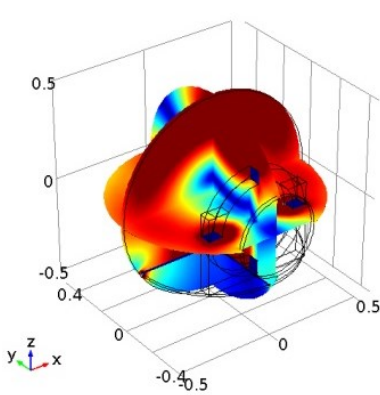

c)

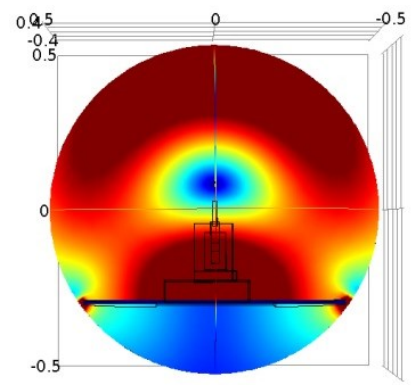

b)

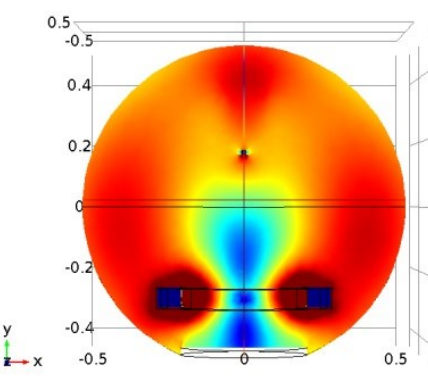

d)
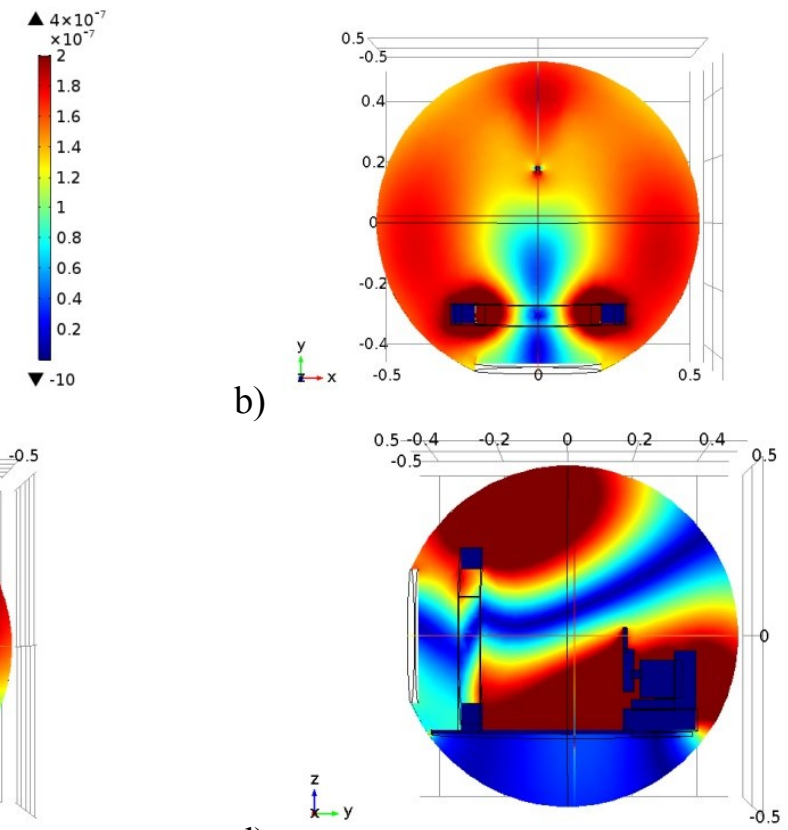

Fig. 3. (a) Simulation of the magnetic flux density distribution (presented in arbitrary units) in the target chamber for the resonant frequency of $287 \mathrm{MHz}$ by inserting the metal plate, lens, metallic lens holder and window.(b)-(d) Top and sides projections of magnetic field inside the target chamber.

The antenna signal is given by the Faraday Law, as follows:

$$
U_{B-P}=-\frac{d}{d t} \Phi_{B}
$$


where $\phi_{B}$ is the magnetic flux through the surface whose boundary is the B-probe's loop. Since we used the same B-probes throughout the experiment, the proportionality factor of the observed induced voltage to the derivative of the flux has been retained during the experiment. It allows us a direct comparison of signals from the B-probes placed elsewhere inside the chamber with corresponding values of $d \phi_{B} / d t$ calculated in those places. An example of the space distribution of $d \phi_{B} / d t$ calculated by the resonant frequency of $287 \mathrm{MHz}$ is shown in Fig. 4.

Although the B-probes can be placed on arbitrary positions, we positioned them around the target at the same height of the focal spot, and at a distance from it of $\sim 35 \mathrm{~cm}$ and $\sim 25 \mathrm{~cm}$, respectively. The first $\mathrm{B}$-probe, $\mathrm{B}_{1}$, was set at $10^{\circ}$ with respect the laser beam direction on the left of the target and the second one, $\mathrm{B}_{2}$, at $45^{\circ}$ on the right of the target (Fig. 1b). Each coil lies on the $y z$-plane.

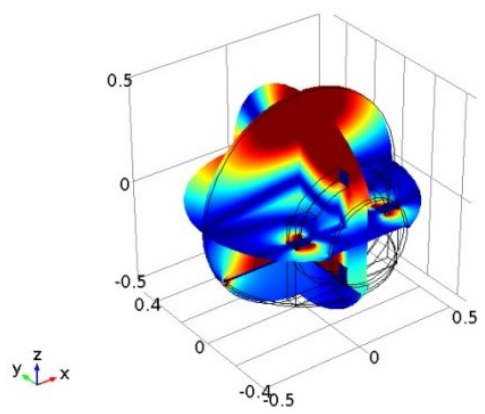

Fig. 4. Space distribution (in arbitrary units) of time derivative of the magnetic flux density calculated by the resonant frequency of $287 \mathrm{MHz}$ in the interaction chamber equipped with basic items.

After ten shots we compared all the spectra of the signals detected by $\mathrm{B}_{1}$ and $\mathrm{B}_{2}$ probes. The analysis shows clearly that only in the frequencies ranges centered in 460 and $780 \mathrm{MHz}$, the voltage induced in $B_{1}$ is clearly higher than that induced in $B_{2}$. Due to the limitation of oscilloscope acquisition, we analyzed the frequencies ranging from 400 to $510 \mathrm{MHz}$, and from 720 to $860 \mathrm{MHz}$. We estimate how much the voltage induced on $\mathrm{B}_{2}$ is less than that induced in $\mathrm{B}_{1}$ in the range of frequencies considered and a comparison of the simulated and the experimental results was done. The simulation presented by the red line in Fig. 5 confirms the observation that the EM field varies strongly inside the interaction chamber with the position of observation, and that the induced voltage is weaker in the position $B_{2}$ in comparison with $B_{1}$. We do not achieve a full agreement of observed data with calculated ones because the simulation can hardly include all the details of all items located inside the interaction chamber. It is also evident that in the interaction chamber there are changes in potentials between different positions and times which are significant for the dynamics of charged particles. These changes are mainly given by the interior design of the interaction chamber.

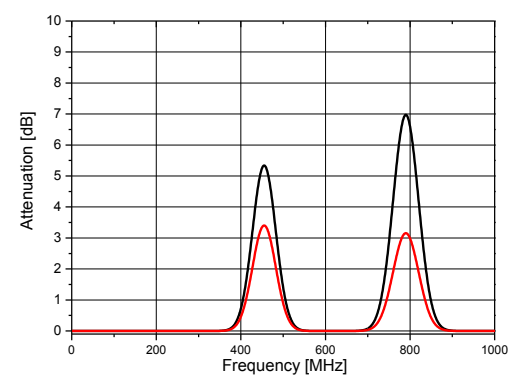

Fig. 5. Comparison of observed (black line) and simulated (red line) attenuation of the spectrum in position $\mathrm{B}_{2}$. 


\section{EMP comparison between RAL and PALS experiments.}

At the Rutherford Appleton Laboratory the Vulcan petawatt laser beam was focused on a $10-\mu \mathrm{m}$ thin $\mathrm{Au}$ foil. The target chamber is a steel parallelepiped with dimensions of $4.6 \times 2.2 \times 2 \mathrm{~m}^{3}$. The B-probe was placed in the target chamber to detect magnetic fields at a distance of $2 \mathrm{~m}$ from the target and the outputs were recorded through flexible double shielded cables by a Tektronix TDS $694 \mathrm{C}-3 \mathrm{GHz}, 10 \mathrm{GS} / \mathrm{s}$ oscilloscope. Our experimental results in terms of frequencies confirmed what was already obtained in [7]. Thus, here we focus our attention on the energy of EMP detected at RAL facility and at the PALS facilities both when the 500J, 350ps PALS laser and the $600 \mathrm{~mJ}, 100 \mathrm{fs}$ TiSa laser were focused in different experiments on the same kind of thick $\mathrm{Cu}$ target inside the PALS chamber. Table 1 presents characteristics of the employed laser systems.

\begin{tabular}{|ccccc|}
\hline Laser & Wavelength & Focus size & Pulse duration & Energy \\
\hline Vulcan & $1053 \mathrm{~nm}$ & $5.27 \mu \mathrm{m}$ & $700 \mathrm{fs}$ & $200-400 \mathrm{~J}$ \\
\hline PALS & $1315 \mathrm{~nm}$ & $80 \mu \mathrm{m}$ & $350 \mathrm{ps}$ & $200-500 \mathrm{~J}$ \\
\hline TiSa-PALS & $808 \mathrm{~nm}$ & $20 \mu \mathrm{m}$ & $100 \mathrm{fs}$ & $0.6 \mathrm{~J}$ \\
\hline
\end{tabular}

Table 1. Main parameters for Vulcan, PALS and TiSa-PALS lasers.

Fig. 6a shows the EMP energy detected with the identical probes in experiments carried out at RAL and PALS facilities versus the energy delivered on targets. Fig. 6b shows the corresponding efficiency defined as the ratio absorbed EMP energy / Laser energy, which gives us the evaluation of the amount of laser energy which is converted into EMP energy detected by our B-probe. The efficiencies also exhibit shot-to-shot fluctuations both at PALS and Vulcan experiments. Nonetheless, the interaction of the 350-ps long pulse of the PALS laser system with the targets is affected by a number of instabilities which destabilize the conversion of laser energy into electron heating including the current flowing from the target to the ground which mainly contributes to the EMP emission $[12,13]$. It is evident that the interaction of the $500-\mathrm{fs}$ Vulcan pulse with target is more stable.

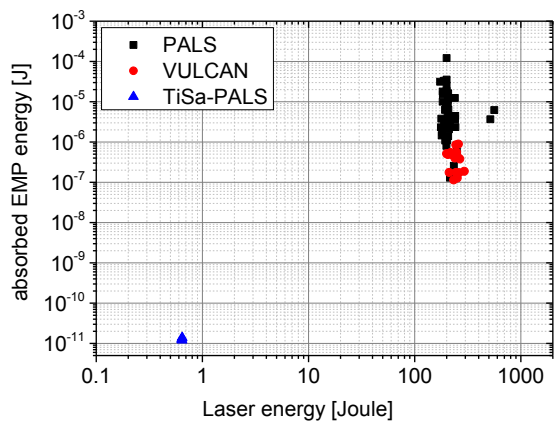

a)

Fig. 6. (a) EMP energy versus laser energy measured at different facilities. (b) Efficiency of EMP generation measured at different facilities in $\%$.

The electromagnetic pulses observed during PALS and Vulcan experiments, are not only caused by various physical processes occurring in different time scales of interaction of 300-ps and 0.7-ps laser pulses with thick and thin targets, resulting in different waveforms of ion fronts, and different gradients of electron temperature and density. The B-probe signals are, in fact, also influenced by the differences in shape and dimension of the interaction chambers, 
the fundamental resonant frequencies and corresponding Q-factors and last but not least the position of B-probes in the chambers. In Fig. 7 the simulated magnetic flux density is shown in the Vulcan target chamber for fundamental resonant frequencies: the dominant mode of a parallelepiped cavity as the Vulcan target chamber, is the TE mode; the simulations shown in Fig. 7 confirmed that the magnetic field resonates at the fundamental frequencies of 76,82 and $101 \mathrm{MHz}$ which were obtained in [7]. The Q-factors at these resonant frequencies are $9.88 \times 10^{4}$, $9.94 \times 10^{4}$ and $12.83 \times 10^{4}$, respectively, while at PALS spherical target chamber, the simulation gave a Q-factor of $8.21 \times 10^{4}$ for the fundamental resonant frequency of $262 \mathrm{MHz}$ (see Fig. 2b).

a)

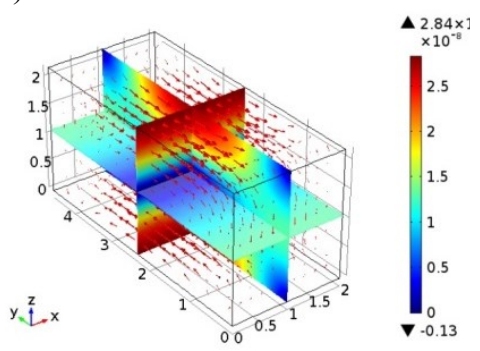

b)

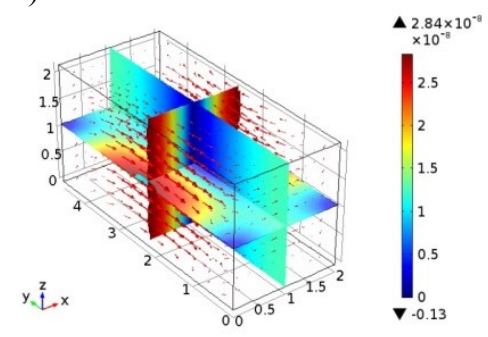

c)

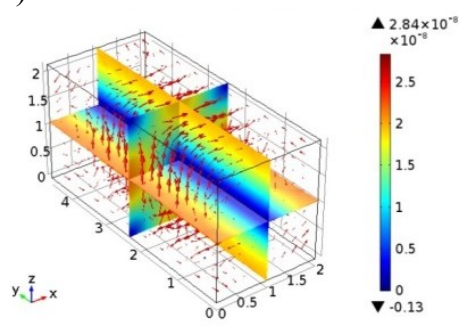

Fig. 7. Fundamental resonant frequencies of $76 \mathrm{MHz}(\mathrm{a}), 82 \mathrm{MHz}(\mathrm{b}), 101 \mathrm{MHz}$ (c) of TE mode in Vulcan target chamber at the RAL Facility. The magnetic field vectors (red arrows) are shown.

\section{Conclusions}

We have carried out several key observations and simulations of the resonating electromagnetic field inside the target chamber which is a helpful tool to predict place-to-place variations in EMP spectra. Significant source of the EMP is the current flowing between the target and the ground which neutralizes a local charge on a target irradiated by a laser. The target holder behaves like an antenna [12] where the current oscillates up to the time of the total neutralization of ionized species moving through the interaction chamber [13]. Nevertheless, presented experiments and simulations show a substantial influence of the shape and size of the interaction chamber on the distribution of the EMP inside the chamber and its emission into the laboratory room. The energy efficiency of the EMP production is dependent on many factors rather than only on the focused laser intensity. Although it was an attempt to simulate only the magnetic fields resonating in the chamber, the results of the numerical simulations are in line with the experimental results, however they cannot completely describe the EMP spectra which are also affected to others factors. Nevertheless, a simple but important basis, for the prediction of EMP parameters and optimization of the diagnostic system design, is presented.

\section{Acknowledgments}

The research leading to these results has received funding from the Czech Science Foundation (Project No. 15-02964S), the Ministry of Education, Youth and Sports of the Czech Republic (ELI-Beamlines reg. No. CZ.1.05/1.1.00/02.0061 and LD14089).

We acknowledge also funding from EPSRC (EP/J002550/1, EP/ L002221/1, EP/ K022415/1 and EP/I029206/1) and Invest Northern Ireland (POC-329). 


\section{References}

[1] J.-L.Dubois et al., Target charging in short-pulse-laser-plasma experiments, Phys. Rev. E 89, 013102 (2014).

[2] J. Cikhardt et al, Measurements of the target current by inductive probe during laser interaction on terawatt laser system PALS, Rev. Sci. Instrum. 85, (2014) 103507.

[3] R.F. Benjamin, G. H. McCall, A. W. Ehler. Measurement of return current in a laser-produced plasma. Phys. Rev. Lett. 42 (1979) 890-893.

[4] C.G. Brown Jr., E. Bond, T. Clancy, S. Dangi, D.C. Eder, W. Ferguson, J.Kimbrough, A.Throop Assessment and mitigation of electromagnetic pulse (EMP) impacts at short-pulse laser facilities. J. Phys. : Conf. Ser. 244 (2010) 032001.

[5] C.G. Brown, J. Ayers, B.Felker, W. Ferguson, J.P. Holder et al. Assessment and mitigation of diagnostic-generated electromagnetic interference at the National Ignition Facility. Rev. Sci. Instrum. 83 (2012) 10D729.

[6] M. De Marco et al., Basic features of electromagnetic pulse generated in a laser-target chamber at 3-TW laser facility PALS, J. Phys.: Conf. Ser. 508 (2014) 012007.

[7] M. J. Mead et al., Electromagnetic pulse generation within a petawatt laser target chamber. Rev. Sci. Instrum. 75, 4225 (2004).

[8] J. L. Remo et al., Atmospheric electromagnetic pulse propagation effects from thick targets in a terawatt laser target chamber", Appl. Optics, vol. 46, pp.6166-6175, 2007

[9] Hyoung Suk Kim. Electromagnetic Waves in Cavity Desig. In: Behaviour of Electromagnetic Waves in Different Media and Structures, Ed.: Ali Akdagli, ISBN: 978-953-307-302-6, InTech 2011, p. 77-100.

[10] S. Ramo, J. R. Whinnery, and T. Van Duzer, Fields and Waves in Communication Electronics (Wiley, New York, 1965), p.541.

[11] http://cdn.comsol.com/documentation/5.0.1.276/IntroductionToCOMSOLMultiphysics.pdf

[12] A. Poyé et al., Physics of giant electromagnetic pulse generation in short-pulse laser experiments. Phys. Rev. E 91, 043106 (2015) .

[13] M. De Marco et al. Electromagnetic pulses produced by expanding laser-produced Au plasma. Nukleonika 10.1515 (2015). 